\title{
Central black hole mass determination for blazars
}

\author{
J. H. Fan, Y. H. Yuan, Y. Huang, Y. Gao, T. X. Hua and Y. Liu \\ ${ }^{1}$ Center for Astrophysics, Guangzhou University, Guangzhou 510006, China
}

\begin{abstract}
In this paper, we used the method to determine the central black mass (M), and the boosting factor $(\delta)$, the propagation angle $(\Phi)$, and the distance along the axis to the site of the $\gamma$-ray production (d) as well for $32 \gamma$-ray loud blazars with available variability timescales. If we take the intrinsic $\gamma$-ray luminosity to be $\lambda$ times the Eddington luminosity, i.e. $L_{\gamma}^{i n}=\lambda L_{E d d}$, then we have following results: the masses of the black hole are in the range of $(0.9 \sim 101) \times 10^{7} M_{\odot}(\lambda=1.0)$ or $(1.30 \sim 153) \times 10^{7} M_{\odot}(\lambda=0.1)$.
\end{abstract}

\section{Introduction}

It is generally believed that the emission of high energy $\gamma$-rays from AGNs depends on $\gamma-\gamma$ pair production process because there are lots of soft photons around the central black hole. In 1995, Becker \& Kafatos, based on the X-ray field of an accretion disk, calculated the $\gamma$-rays optical depth, and found that the $\gamma$-rays should escape preferentially along the symmetric axis of the disk, due to the strong angular dependence of the pair production cross section. The phenomenon of $\gamma-\gamma$ 'focusing' is related to the main issue of $\gamma-\gamma$ transparency, which represents a minimum distance between the central black hole and the site of $\gamma$-ray production (Fan 2005, Cheng et al. 1999). Therefore, the $\gamma$-rays are focused in a solid angle, $\Omega=2 \pi(1-\cos \Phi)$, so the apparent observed luminosity can be expressed as: $L_{\gamma}=\Omega D^{2} F_{\gamma}(1+z)^{\alpha_{\gamma}-1}$, here $F_{\gamma}$ is the observed $\gamma$-ray energy flux, D the luminosity distance, $\mathrm{z}$ the redshift, $\alpha_{\gamma}$ the $\gamma$-rays spectral index. Because the observed $\gamma$-rays from blazars demand that the jet is almost pointing to us, so the optical depth $\tau$ is not greater than unity. The $\gamma$-rays come from a solid angle, $\Omega$, instead of being isotropic. So, both the beaming and absorption effects must be considered when the properties of a $\gamma$-rays blazars are discussed. The optical variability supplies us with some information about the $\gamma$-ray emission region. All these considerations give us a method to estimate the basic parameters of $\gamma$-rays loud blazars, including the central black hole mass $(M)$, the boosting factor $(\delta)$, the propagation angle $(\Phi)$ and the distance along the axis to the site of the $\gamma$-ray production $(d)$.

\section{Method}

In this part, from our previous papers by Cheng, Fan, Zhang (1999) and Fan (2005) who obtained the optical depth expression based on the work by Becker \& Kafatos (1995), we have four equations for the four parameters:

$$
\begin{gathered}
\frac{d}{R_{g}}=1.73 \times 10^{3} \frac{\triangle T_{D}}{1+z} \delta M_{7}^{-1} \\
L_{\text {iso }}^{45}=\frac{2.52 \lambda \delta^{\alpha_{\gamma}+4}}{(1-\cos \Phi)(1+z)^{\alpha_{\gamma}-1}} M_{7}
\end{gathered}
$$


Table 1. Central Black Hole Masses for Blazars

\begin{tabular}{|c|c|c|c|c|c|c|c|c|}
\hline Name & $\begin{array}{c}\log \left(\frac{M}{M_{\odot}}\right) \\
\lambda=0.1\end{array}$ & $\begin{array}{c}\log \left(\frac{M}{M_{\odot}}\right) \\
\lambda=1.0\end{array}$ & Name & $\begin{array}{c}\log \left(\frac{M}{M_{\odot}}\right) \\
\lambda=0.1\end{array}$ & $\begin{array}{c}\log \left(\frac{M}{M_{\odot}}\right) \\
\lambda=1.0\end{array}$ & Name & $\begin{array}{c}\log \left(\frac{M}{M_{\odot}}\right) \\
\lambda=0.1\end{array}$ & $\begin{array}{c}\log \left(\frac{M}{M_{\odot}}\right) \\
\lambda=1.0\end{array}$ \\
\hline $0202+149$ & 7.9 & 8.08 & $0827+243$ & 6.94 & 7.12 & $1604+159$ & 8.4 & 8.58 \\
\hline 0208-512 & 9.01 & 9.19 & $0836+710$ & 7.8 & 7.97 & $1606+106$ & 8.32 & 8.48 \\
\hline $0219+428$ & 8.47 & 8.65 & OJ287 & 7.12 & 7.31 & $1611+343$ & 8.2 & 8.37 \\
\hline $0235+164$ & 8.67 & 8.86 & $0906+430$ & 8.25 & 8.42 & $1622-297$ & 7.9 & 8.07 \\
\hline $0234+285$ & 8.24 & 8.41 & $0917+449$ & 7.87 & 8.06 & $1633+382$ & 8.36 & 8.54 \\
\hline 0336-019 & 7.55 & 7.73 & $0954+556$ & 8.39 & 8.56 & Mrk501 & 8.02 & 8.22 \\
\hline 0420-014 & 8.41 & 8.58 & $0954+658$ & 8.6 & 8.77 & NRAO 530 & 8.35 & 8.52 \\
\hline NRAO190 & 7.53 & 7.7 & $1011+496$ & 8.35 & 8.53 & $1739+522$ & 8.24 & 8.41 \\
\hline B0454-234 & 8.24 & 8.4 & $1055+567$ & 8.37 & 8.54 & B1741-038 & 8.21 & 8.38 \\
\hline J0454-463 & 8.27 & 8.43 & Mrk 421 & 7.28 & 7.49 & $1830-210$ & 8.32 & 8.49 \\
\hline В0458-020 & 8.82 & 8.99 & B1127-145 & 7.77 & 7.94 & $1933-400$ & 8.32 & 8.48 \\
\hline J0506-612? & 8.24 & 8.41 & $1156+295$ & 8.2 & 8.38 & $2005-489$ & 8.2 & 8.34 \\
\hline $0528+134$ & 8.35 & 8.52 & $1219+285$ & 7.17 & 7.37 & $2032+107$ & 8.3 & 8.44 \\
\hline B0521-365 & 8.67 & 8.83 & $1222+216$ & 8.38 & 8.55 & $2052-474$ & 8.19 & 8.38 \\
\hline B0537-286 & 7.79 & 7.97 & $3 \mathrm{C} 273$ & 8.13 & 8.3 & 2155-304 & 7.55 & 7.71 \\
\hline 0537-441 & 8.33 & 8.5 & B1229-021 & 8.24 & 8.4 & BL Lac & 8.02 & 8.17 \\
\hline $0716+714$ & 7.51 & 7.68 & $3 \mathrm{C} 279$ & 8.65 & 8.83 & CTA 102 & 8.4 & 8.57 \\
\hline $0735+178$ & 8.35 & 8.51 & $1331+170$ & 8.16 & 8.33 & 3C 454.3 & 7.21 & 7.4 \\
\hline $0804+499$ & 8.04 & 8.22 & B1334-127 & 8.2 & 8.36 & $2356+196$ & 8.25 & 8.42 \\
\hline $0829+046$ & 8.55 & 8.71 & B1510-089 & 8.56 & 8.73 & & & \\
\hline
\end{tabular}

$$
9 \times \Phi^{2.5}\left(\frac{d}{R_{g}}\right)^{-\frac{2 \alpha_{X}+3}{2}}+k M_{7}^{-1}\left(\frac{d}{R_{g}}\right)^{-2 \alpha_{x}-3}=1
$$

$22.5 \Phi^{1.5}(1-\cos \Phi)-9 \times \frac{2 \alpha_{X}+3}{2 \alpha_{\gamma}+8} \Phi^{2.5} \sin \Phi-\frac{2 \alpha_{X}+3}{\alpha_{\gamma}+4} k M_{7}^{-1} A^{-\frac{2 \alpha_{X}+3}{2}}(1-\cos \Phi)^{-\frac{\alpha_{X}+3}{2 \alpha_{\gamma}+8}} \sin \Phi=0$

For a source with available X-ray, $\gamma$-ray data, and with data on short time scales, $M_{7}$, $\delta, \mathrm{d}$, and $\Phi$, can be derived from the upper four equation, here $R_{m s}=6 R_{g}, R_{0}=30 R_{g}$, $\omega=3$ (a two-temperature disk) and $E_{\gamma}=1 G e V$ have been used. The results are shown in Table 1. The results are also compared with those by Barth et al. (2003); Rieger \& Mannheim (2003); Shen et al. (2006); Wang et al. (1996); Woo \& Urry (2003). Refer to our full paper for the detail consideration (Fan et al. 2007 in preparation).

\section{Acknowledgements}

This work is supported by NSFC $(10573005,10633010)$ and the 973 project (2007CB815405). We also thank the financial support from the Guangzhou Education Bureau and Guangzhou Science and Technology Bureau.

\section{References}

Barth, A. J., Ho, L. C., \& Sargent, W. L. W. 2003, ApJ, 583:134-144

Becker, P., \& Kafatos, M. 1995, ApJ, 453, 83

Cheng, K. S., Fan, J. H., \& Zhang, L. 1999, A\&A, 352, 32

Fan, J. H. 2005, A\&A, 436, 799

Rieger, F. M. \& Mannheim, K. 2003, A\&A, 397, 121

Shen, Z. Q. 2006, in the Central Engine of Active Galactic Nuclei, Xi'an, China, 16-21 Oct., 2006

Wang, J. M., Chen, Y. M., \& Hu, C. 2006, ApJ, 637, 41

Woo, J. H. \& Urry, C. M. 2002, ApJ, 579, 530 